\title{
NOUVELle
}

\section{L'entose, mort cellulaire par cannibalisme entre cellules tumorales}

Arnaud A. Mailleux, Michael Overholtzer, Joan S. Brugge
Harvard Medical School, Cell Biology Department, 240 Longwood Avenue, Boston MA 02115, États-Unis. Joan_Brugge@hms.harvard.edu
> La mort cellulaire joue un rôle clé dans le développement de nombreux organes et dans l'homéostasie tissulaire chez l'adulte. Un dérèglement de l'équilibre entre la prolifération et la mort cellulaires peut conduire au développement de maladies dégénératives par un excès de mort cellulaire, ou à l'émergence de cancers en cas de déficit. Les cellules peuvent déclencher leur autodestruction en réponse à un stress intracellulaire ou un signal de mort extracellulaire. Ce processus de mort cellulaire physiologique appelé apoptose (ou suicide cellulaire) est génétiquement contrôlé et fut particulièrement étudié ces dernières années [1].

Par exemple, le détachement de cellules épithéliales de la matrice extracellulaire sous-jacente déclenche leur élimination par une forme d'apoptose appelée anoikis [2] (du grec «sans-abri»). L'anoikis est ainsi induite par la perte de l'engagement des intégrines membranaires qui ancrent les cellules aux protéines de la matrice extracellulaire. Les intégrines sont habituellement connectées aux microfilaments d'actine par leur domaine intracellulaire. Les cellules cancéreuses sont généralement résistantes à la mort induite par anoikis. Cette résistance leur conférerait potentiellement la capacité de survivre au voyage en dehors de la tumeur primaire et de former ainsi des métastases.

D'autres processus de mort non-apoptotique, comme la nécrose cellulaire ou l'autophagie par exemple [3], peuvent parfois agir en synergie avec l'apoptose pour occire efficacement une cellule et peuvent aussi compenser une apoptose déficiente $[4,5]$.

\section{Mise en évidence}

et caractéristiques de l'entose

Récemment, un autre processus de mort cellulaire alternatif a été mis en évidence lorsque des cellules épithéliales perdent contact avec leur substrat in vitro [6]. Ainsi, des cellules de lignées épithéliales d'origine mammaire peuvent aussi former en suspension des agrégats où des cellules se retrouvent complètement encapsulées dans des vacuoles au sein de cellules voisines (Figure 1). Ce processus nommé « entose», du grec entos («dedans ») se déroulait en parallèle de l'anoikis dans ce modèle in vitro [6]. À première vue, la concomitance de ces deux processus pourrait suggérer que ce phénomène d'internalisation correspond à la phagocytose de cellules mortes en anoikis par leurs voisines encore en vie. Au cours de l'apoptose, la cellule mourante émet des signaux. En particulier, elle expose sur le feuillet externe de la membrane plasmique des résidus phosphatidylsérine (PS), un phospholipide normalement situé sur son feuillet interne, qui permettront sa reconnaissance, sa phagocytose et son élimination par les macrophages et d'autres

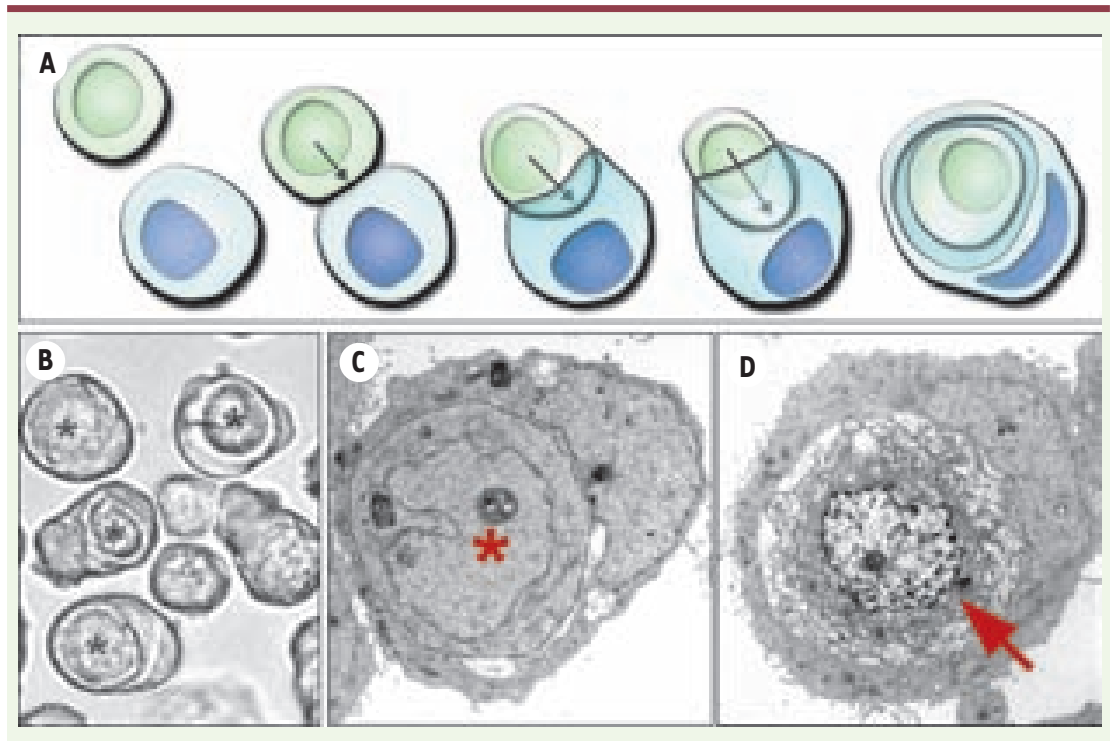

Figure 1. L'entose cellulaire. A. Représentation schématique de l'entose : une cellule (en vert) va envahir activement (flèches) une autre cellule (en bleu) et finir encapsulée dans cette dernière. B. Figures d'entose (astérisques) dans une culture en suspension de cellules MCF-10A d'origine mammaire. $C, D$. Images en microscopie électronique à transmission de figures d'entose formées par les cellules MCF-7 en suspension. Noter que la cellule internalisée (astérisque rouge et délimitée par les pointillés) en ( $C$ ) est vivante alors que celle montrée en ( $D$ ) est en cours de dégradation (flèche rouge) par un processus de mort cellulaire non-apoptotique. 
type cellulaires dont les cellules épithéliales mammaires [7-9]. Au cours de l'entose, la cellule internalisée ne présente pas les marqueurs caractéristiques de l'apoptose tant au niveau morphologique que biochimique. En particulier, ces cellules n'exposent pas de résidus PS sur leur feuillet membranaire extérieur. De plus, l'inhibition de l'apoptose en général n'avait aucun effet sur l'entose de ces cellules en suspension. Contrairement à la phagocytose de corps apoptotiques, la cellule internalisée est donc parfaitement vivante au cours de l'entose. Des divisions cellulaires sont parfois observées dans ces vacuoles confirmant la bonne santé de la cellule internalisée.

D'un point de vue mécanistique (Figure $1 A$ ), l'entose nécessite la formation de jonctions adhérentes intercellulaires faisant intervenir les protéines adhésives de la famille des cadhérines. II est probable que lors de la perte de l'engagement des intégrines, les cellules essayent de s'attacher sur le seul substrat disponible: une autre cellule. II s'ensuivrait un processus d'internalisation nécessitant l'intervention du cytosquelette d'actine/myosine sous la dépendance de la protéine GTPase Rho et de son effecteur Rock, une protéine kinase. La voie de signalisation de la protéine GTPase Rho joue un rôle central dans le contrôle de la dynamique du cytosquelette d'actine/myosine [10]. Un déséquilibre des forces contractiles générées par les jonctions adhérentes entre les deux cellules pourrait ainsi expliquer l'internalisation d'une des cellules. Paradoxalement, l'activation de la voie Rho/Rock n'est nécessaire que pour la cellule internalisée suggérant que la cellule hôte est passive de ce point de vue. En conclusion, la cellule « entosée » est bien vivante, active et va littéralement envahir l'autre cellule (Figure 1).

À la suite de leur intrusion dans la cellule hôte, les cellules internalisées sont encapsulées dans une vacuole et sont généralement dégradées par des enzymes d'origine lysosomale (Figure IC$D, 2 A)$. Ce processus de mort est médié par les lysosomes et ne semble pas apoptotique. La cellule internalisée est ainsi cannibalisée et digérée vivante dans les entrailles de l'autre cellule.
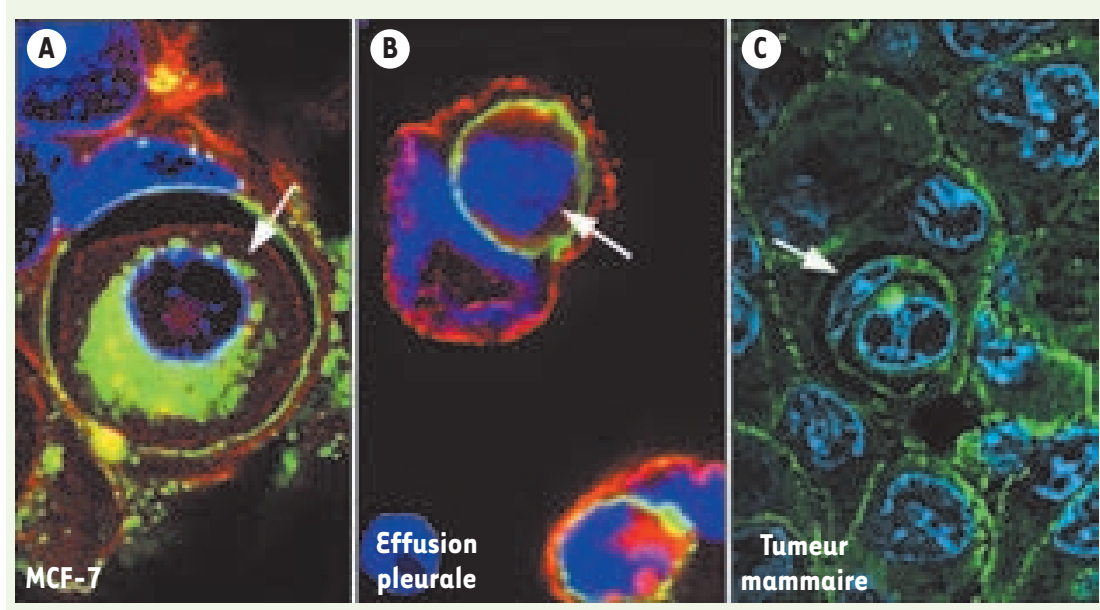

Figure 2. Images d'entose en microscopie confocale. A. Images d'entose dans des agrégats de cellules mammaires MCF-7 cultivées en suspension in vitro. Le marquage pour la $\beta$-caténine (rouge) marque les contours cellulaires et Lampl (vert) marque les lysosomes (bleu : DAPI). B. Images d'entose dans des effusions pleurales de patients atteints de cancer du sein. Le marquage en rouge pour MUC- 1 identifie les cellules épithéliales et $\beta$-caténine en vert les contacts entre la cellule internalisée et la cellule cellule hôte (bleu: DAPI). C. Images d'entose au sein de tumeurs solides d'origine mammaire (vert: $\beta$-caténine; bleu : DAPI).
Il faut aussi noter qu'un petit nombre de cellules internalisées sont relâchées par la cellule hôte ou réussissent à s'échapper.

\section{Pertinence de l'entose in vivo}

L'entose n'est pas restreinte uniquement aux cellules d'origine mammaire (Figure 2A). Par exemple, ce processus a été observé in vitro dans d'autres lignées cellulaires d'origine ovarienne, endothéliale ou mésothéliale cultivées en suspension [6]. Au premier abord, il semblerait possible que l'entose ne soit qu'un artefact observé in vitro lorsque des cellules sont cultivées en suspension. Néanmoins, d'un point morphologique, l'entose ressemble aux figures de cannibalisme cellulaire décrites en cytologie où une cellule tumorale se retrouve internalisée dans une autre cellule cancéreuse [11]. Ce phénomène est observé dans des tumeurs solides et dans les effusions pleurales de certains patients atteints de métastases [11] (Figure 2B-C). Une étude plus approfondie [6] montre que ces figures de cannibalisme entre cellules tumorales se caractérisent aussi par la formation de jonctions adhérentes en l'absence d'attachement avec la matrice et ce même dans les tumeurs solides. De plus, la cellule internalisée était souvent digérée par les lysosomes dans la cellule hôte. Ainsi, l'entose semble correspondre au phénomène de cannibalisme entre cellules cancéreuses observé in vivo dans certains types de tumeurs. Il existe aussi d'autres formes de cannibalisme cellulaire où des cellules de mélanome malin peuvent internaliser des lymphocytes T [12] par exemple.

Cependant, quel est l'impact de l'entose sur la croissance d'une tumeur? Le fait que des cellules s'éliminent entre elles suggère que l'entose serait un processus suppresseur de tumeur. De plus, l'inhibition de l'entose dans un modèle in vitro de tumorigenèse augmente la taille de colonies formées par des cellules cancéreuses [6]. 


\section{En conclusion}

L'entose se caractérise par l'invasion d'une cellule par une autre menant à la destruction de la cellule internalisée. Ce processus est observé in vivo dans des échantillons provenant de patients atteints de cancer et semble ralentir la croissance de la masse tumorale in vitro. Cependant les questions de la régulation de l'entose et son impact sur la croissance tumorale in vivo restent ouvertes. $\diamond$

Entosis, a cell death process related to cell cannibalism between tumor cells

\section{RÉFÉRENCES}

1. Jacobson MD, Weil M, Raff MC. Programmed cell death in animal development. Cell $1997 ; 88: 347-54$.

2. Frisch SM, Francis H. Disruption of epithelial cellmatrix interactions induces apoptosis. J Cell Biol $1994 ; 124: 619-26$.

3. Codogno $P$. Les gènes ATG et la macro-autophagie. Med Sci (Paris) $2004 ; 20: 734-6$.

4. Debnath J, Baehrecke $\varepsilon H$, Kroemer G. Does autophagy contribute to cell death? Autophagy $2005 ; 1:$ :66-74.

5. Mailleux AA, Overholtzer M, Schmelzle T, et al. BIM regulates apoptosis during mammary ductal morphogenesis, and its absence reveals alternative cell death mechanisms. Dev Cell 2007 ; 12 : 221-34.

6. Overholtzer M, Mailleux AA, Mouneimne G, et al. A nonapoptotic cell death process, entosis, that occurs by cell-in-cell invasion. Cell 2007 ; 131: 966-79.
7. Fadok VA, Bratton DL, Rose DM, et al. A receptor for phosphatidylserine-specific clearance of apoptotic cells. Nature $2000 ; 405: 85-90$.

8. Monks J, Rosner D, Geske FJ, et al. Epithelial cells as phagocytes : apoptotic epithelial cells are engulfed by mammary alveolar epithelial cells and repress inflammatory mediator release. Cell Death Differ 2005 ; 12 : 107-14

9. Chimini G. Le récepteur des phosphatidylsérines, une arlésienne qui nous surprend toujours. Med Sci (Paris) $2004 ; 20: 501-2$.

10. Arthur WT, Noren NK, Burridge K. Regulation of Rho family GTPases by cell-cell and cell-matrix adhesion. Biol Res 2002 ; 35 : 239-46.

11. Abodief WT, Dey P, Al-Hattab O. Cell cannibalism in ductal carcinoma of breast. Cytopathology 2006 ; $17: 304-5$.

12. Lugini L, Matarrese $P$, Tinari A, et al. Cannibalism of live lymphocytes by human metastatic but not primary melanoma cells. Cancer Res 2006 ; $66: 3629-38$.

\section{NOUVELle}

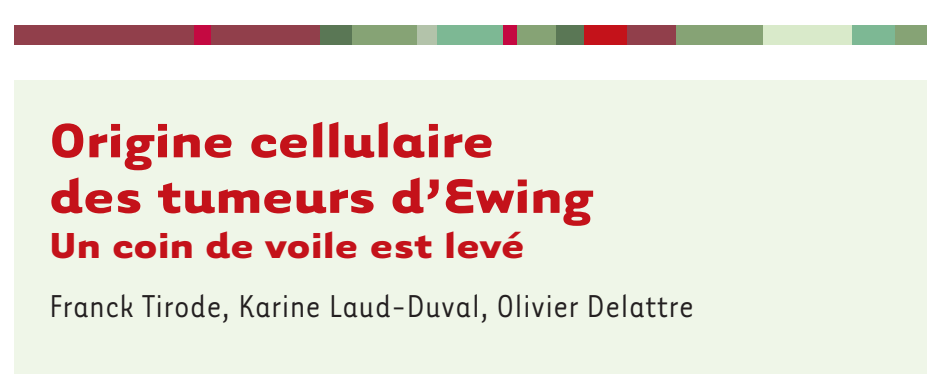

$>$ La tumeur d'Ewing, seconde tumeur de l'os par ordre de fréquence chez les adolescents et les jeunes adultes, présente une translocation chromosomique caractéristique fusionnant la partie 5' du gène EWS à la partie 3' d'un membre de la famille ETS. La fusion la plus commune, $\varepsilon W S-F L I-1$, résulte d'une translocation entre les chromosomes 11 et 22 [1]. Le rôle causal de cette fusion dans le développement tumoral a été clairement documenté. La compréhension des mécanismes intimes de cette transformation cellulaire repose très schématiquement d'une part sur l'élucidation du rôle biologique de la protéine EWS-FLI-1, et en particulier sur l'identification des principaux gènes cibles de ce facteur de transcription aberrant, et d'autre par sur la caractérisation précise du contexte cellulaire au sein duquel s'exerce cet effet.
Nature de la cellule cible à l'origine des tumeurs d'Ewing : les multiples hypothèses

D'un point de vue histologique, ces tumeurs sont caractérisées par de petites cellules rondes indifférenciées, possédant un petit cytoplasme, intriquées dans un stroma intercellulaire épars. Ces caractéristiques phénotypiques sont peu informatives sur la cellule à l'origine des tumeurs. II y a 86 ans, James Ewing, auteur de la première description de cette tumeur [2], proposait une origine endothéliale. Depuis lors, plusieurs hypothèses alternatives ont été proposées. L’hypothèse neurale est fondée sur l'observation que la fusion EWS-FLI-1 caractérise certes des tumeurs très indifférenciées mais aussi des tumeurs neuroectodermiques primitives qui expriment des marqueurs spécifiques du tissu neural. De plus,
Inserm U830, Institut Curie, 26, rue d'Ulm, 75248 Paris, France. olivier.delattre @curie.fr

des cellules d'Ewing cultivées in vitro en présence d'une combinaison de facteurs de différenciation spécifique, forment des structures semblables à des neurites et expriment certains marqueurs caractéristiques de la lignée neurale $[3,4]$. Une autre hypothèse, fondée sur l'expression par les cellules d'Ewing de certaines protéines des jonctions serrées (comme ZO-l ou la claudine) évoque une origine cellulaire épithéliale [5]. Enfin, des études récentes menées chez la souris ont soulevé l'hypothèse mésenchymateuse. L'équipe de Suzan Backer a ainsi pu mettre en évidence que l'expression d'EWS-FLI-I dans des cellules stromales osseuses bloquait leur différenciation terminale dans les voies adipocytaire et ostéocytaire [6]. Puis les équipes de Robert Ilaria et d'Ivan Stamenkovic ont montré que des cellules stromales osseuses exprimant EWS- 